\title{
Effectiveness of eLearning and blended modes of delivery of Mental Health First Aid training in the workplace: randomised controlled trial
}

Nicola J. Reavley ${ }^{1 *}$ (D), Amy J. Morgan ${ }^{1}$, Julie-Anne Fischer', Betty Kitchener², Nataly Bovopoulos² and Anthony F. Jorm ${ }^{1}$

\begin{abstract}
Background: The aim of the WorkplaceAid study was to compare the effects of eLearning or blended (eLearning plus face-to-face course delivery) Mental Health First Aid (MHFA) courses on public servants' knowledge, stigmatising attitudes, confidence in providing support and intentions to provide support to a person with depression or post-traumatic stress disorder (PTSD).

Methods: A randomized controlled trial was carried out with 608 Australian public servants. Participants were randomly assigned to complete an eLearning MHFA course, a blended MHFA course or Red Cross eLearning Provide First Aid (PFA) (the control). The effects of the interventions were evaluated using online questionnaires pre- and post-training. The questionnaires centred around vignettes describing a person meeting the criteria for depression or PTSD. Primary outcomes were mental health first aid knowledge and desire for social distance. Secondary outcomes were recognition of mental health problems, beliefs about treatment, helping intentions and confidence and personal stigma. Feedback on the usefulness of the courses was also collected.

Results: Both the eLearning MHFA and blended MHFA courses had positive effects compared to PFA eLearning on mental health first aid knowledge, desire for social distance, beliefs about professional treatments, intentions and confidence in helping a person and personal stigma towards a person with depression or PTSD. There were very small non-significant differences between the eLearning MHFA and blended MHFA courses on these outcome measures. However, users were more likely to highly rate the blended MHFA course in terms of usefulness, amount learned and intentions to recommend the course to others.
\end{abstract}

Conclusions: The blended MHFA course was only minimally more effective than eLearning MHFA in improving knowledge and attitudes. However, course satisfaction ratings were higher from participants in the blended MHFA course, potentially leading to greater benefits in the future. Longer-term follow-up is needed to explore this.

Trial registration: ACTRN12614000623695 registered on 13/06/2015 (prospectively registered).

\footnotetext{
* Correspondence: nreavley@unimelb.edu.au

${ }^{1}$ Centre for Mental Health, Melbourne School of Population and Global

Health, University of Melbourne, 207 Bouverie Street, Carlton, VIC 3010,

Australia

Full list of author information is available at the end of the article
}

(c) The Author(s). 2018 Open Access This article is distributed under the terms of the Creative Commons Attribution 4.0 International License (http://creativecommons.org/licenses/by/4.0/), which permits unrestricted use, distribution, and reproduction in any medium, provided you give appropriate credit to the original author(s) and the source, provide a link to the Creative Commons license, and indicate if changes were made. The Creative Commons Public Domain Dedication waiver (http://creativecommons.org/publicdomain/zero/1.0/) applies to the data made available in this article, unless otherwise stated. 


\section{Introduction}

Because of the high prevalence of mental disorders, members of the public are very likely to have contact with people with mental health problems (which includes those with diagnosed disorders or subclinical symptoms) and can play a valuable role in providing support $[1,2]$. It can therefore be argued that the public need knowledge and skills to provide help to people with mental health problems. This help, also known as 'mental health first aid', can be defined as the help provided to a person developing a mental health problem, experiencing a worsening of a mental health problems or in a mental health crisis (e.g. at immediate risk of suicide) [3]. The first aid is given until appropriate professional treatment is received or until the crisis resolves. The Mental Health First Aid (MHFA) training course teaching these skills was developed in Australia in 2000 [4]. It involves 12 hours of face-to-face instruction and gives an overview of the most common and disabling mental health problems, introduces a five-component action plan and then applies these actions to help people with problems of depression, anxiety disorders, psychosis and substance use disorder as well as crisis situations including suicidal behaviours, panic attacks, traumatic events, aggressive behaviour, and drug overdose [5].

Since its inception, MHFA training has expanded rapidly; there are now 1500 accredited MHFA instructors in Australia who have trained over 500,000 adults. It operates in over 20 countries and 2 million people have been trained globally [6]. Evaluation studies were first carried out in Australia and have now been undertaken in other countries. A recent meta-analysis conducted by Morgan et al. [7] included 18 randomised controlled trials that evaluated the effectiveness of MHFA in a range of settings. MHFA training led to improved mental health first aid knowledge with effects persisting up to 12 months after training. There were also moderate improvements in recognition of mental disorders and beliefs about effective treatments and and small reductions in stigmatising attitudes (ds 0.08-0.15). Perceived confidence in helping a person with a mental health problem and intentions to provide first aid were also seen post-training and persisted for up to 6 months later. There were small improvements in the amount of help provided to a person with a mental health problem at follow-up $(d=0.23)$ although impact of MHFA on the quality of help offered was not clear.

Eight of the studies included in the meta-analysis mentioned above were conducted in workplace settings, which is increasingly seen as an important setting for mental health education and training, not only to address mental health problems caused by work, but also to address mental health problems that arise as a result of other factors but that may become apparent in the workplace or be exacerbated by poor working conditions $[8,9]$. Growing evidence suggests that workplace interventions may produce improvements in mental health literacy [10] and depression and anxiety symptoms [9, 11 ] as well as reductions in stigma [10].

In the context of the growing popularity of eLearning, an electronic version of MHFA was developed and evaluated in a randomised controlled trial (RCT) conducted in 2009 involving 262 members of the public, who were randomly assigned to complete the eLearning course, read a printed MHFA manual or be in a wait-list control group [12]. Participants were sent weekly emails for four weeks to prompt them to continue participating. The impact of the interventions was evaluated using online questionnaires administered at baseline, post-training and at 6-months follow-up. Both eLearning and the printed manual were superior to wait list in increasing knowledge, reducing stigmatising attitudes and increasing confidence. eLearning showed greater effects than wait-list or manual in reducing stigmatising attitudes to a person with schizophrenia and also in improving first-aid actions taken.

As with other intervention settings, recent years have seen an increase in online interventions in the workplace. eLearning may be particularly appropriate to workplace settings, where it is not always easy to roster staff to attend training simultaneously, particularly for longer courses such as face-to-face MHFA training which is 12 hours long and requires four sessions of three hours each. Other groups of people for whom attendance is difficult, are those living in remote areas or who are distant from where courses are offered, shift workers, and people who have family commitments which make it difficult to attend for this length of time. However, one of the disadvantages of the eLearning format is that it lacks the group interaction, discussion and questions that are part of a face-to-face course.

One approach to training that aims to maximise the convenience of eLearning with the interactivity of face-to-face learning, is that of blended learning. Common in the field of health professional education, blended learning has been shown to be more effective or at least as effective as non-blended instruction for knowledge acquisition [13]. However, authors of a recent meta-analysis that included 56 studies found large heterogeneity across studies and noted that their conclusions should be treated with caution [13]. Another meta-analysis of eLearning trials covered 96 studies of 168 training courses [14] and focused on comparisons between face-to-face instruction and blended learning or eLearning. Results showed that, for declarative knowledge (memory of facts and principles taught), effects were larger for blended learning (Cohen's $d=0.35(95 \%$ CI $0.29-0.39)$ ) than eLearning only $(d=0.15$ (95\% CI 
0.11-0.19)). The findings were similar for procedural knowledge (information about how to perform a task or action): $\mathrm{d}=0.53$ (95\% CI $0.34-0.70)$ vs $\mathrm{d}=-0.07(95 \%$ CI -0.20-0.06). This meta-analysis also looked at learner satisfaction with the mode of instruction and found a very small effect in favour of online compared to blended learning. However, the studies included in these reviews focussed specifically on its effectiveness with adults acquiring knowledge directly relevant to their current or future employment. There has been very little research into the effectiveness of mode of instruction for interventions targeted to the general public where the content does not relate to employment. Furthermore, a review of anti-stigma interventions found no difference in effects between studies with internet or non-internet delivery of content, but head-to-head comparisons of delivery mode are rare [15]. No prior studies have compared the effectiveness of MHFA training when delivered in an eLearning versus blended mode.

The principal aim of this study was to compare the effects of eLearning or blended (eLearning plus face-toface course delivery) MHFA training on knowledge, stigmatising attitudes, confidence in providing support and intentions to provide support to a person with depression or PTSD. Participants were members of the public service in Victoria and the Australian Capital Territory (ACT), Australia.

Study hypotheses were as follows:

1. Compared to eLearning Provide First Aid (PFA) training (the control), MHFA training, whether delivered in eLearning or blended modes, will result in higher mental health first aid knowledge, lower stigmatizing attitudes, and greater confidence in providing support to a person developing a mental health problem or in a mental health crisis.

2. Compared to eLearning MHFA training, blended MHFA training will result in higher mental health first aid knowledge, lower stigmatizing attitudes, and greater confidence in providing support to a person developing a mental health problem or in a mental health crisis.

3. Compared to eLearning MHFA training, blended MHFA training will be rated higher on satisfaction than eLearning MHFA training.

\section{Methods}

\section{Study design}

The WorkplaceAid study was a parallel group RCT with participants randomized to eLearning MHFA, blended MHFA or PFA eLearning in a 1:1:1 ratio. The trial was registered with the Australian and New Zealand Clinical Trials Registry (ACTRN12614000623695, registered on 13 June 2014). PFA eLearning was chosen as a control intervention as it deals with health problems that may occur in the workplace, uses a similar mode of administration to the eLearning MHFA training and involves a similar time commitment.

\section{Participants}

Australian public servants who were aged 18 and over and did not hold a current certificate in either MHFA or PFA were eligible for the study. Initially, state government employees in the state of Victoria, Australia, were eligible to participate. In 2016, recruitment was opened to Commonwealth government employees in the ACT in order to counteract a slow-down in recruitment in the Victorian public service after the 2014 state election and subsequent department restructure. Participants were informed about the study through flyers put up in offices, articles in newsfeed items on the staff intranet and at staff wellbeing events. Potential participants were asked to register online by going to a trial website, which contained a Participant Information Sheet, an 'I accept' checkbox and a link to the baseline SurveyMonkey questionnaire. After completion of this questionnaire, participants were randomized to one of the three groups listed above and given instructions on how to access the relevant course. Participants were advised that they could complete the training during work hours, with their manager's approval, avoiding busy times at work.

\section{Randomization}

Randomization was carried out by a random integer generator programmed on the trial website to give values of $1=$ eLearning MHFA; $2=$ Blended MHFA and 3 = PFA eLearning (see http://php.net/manual/en/function.rand.php). Allocation was concealed as randomization was computer generated. Blinding was not possible due to the nature of the interventions.

\section{Interventions \\ eLearning MHFA}

This intervention was a 6-hour eLearning MHFA course accessed via the MHFA Australia online portal. The course teaches a 5-component action plan for responding to developing mental health problems such as depression, anxiety problems, psychosis and substance use problems, and crises including suicidal thoughts and behaviours, non-suicidal self-injury, panic attacks, traumatic events, severe psychotic states, severe effects from alcohol misuse, severe effects from drug misuse, and aggressive behaviours. The eLearning MHFA course comprised 5 modules which needed to be completed in sequence, with a quiz at the end of each: Introduction to mental health; Depression; Anxiety Problems; Psychosis and Substance Use Problems. Each module included 
interactive content on case studies (click on a picture or table to answer). The course also includes audio and video content depicting stories of lived experience and demonstrating how to provide mental health first aid, following by questions or other activities.

The online content was tailored to incorporate information on resources and help-seeking pathways of specific relevance to the relevant public service (e.g. Employee Assistance Program (EAP) providers). An accompanying hard copy MHFA manual (which was regularly referred to in the eLearning course) was posted to participants when they registered for the course [5]. Participants received weekly automated emails for 6 weeks to pace them through the material. Monthly reports were extracted to monitor course progress. On course completion, an automatic email was sent to the trial manager to flag completion and another was sent to the participant containing a link to the post-course questionnaire.

\section{Blended MHFA}

This intervention included the 6-hour eLearning course described above plus a 4-hour face-to-face session, which reviewed the contents of the online course through quizzes and discussion. It also included case studies and role plays to give participants more experience in applying the MHFA Action Plan in different situations and settings. Group training was completed within 3 months of online course completion. In Melbourne and Ballarat, Victoria, two MHFA Instructors ran 16 courses individually, while one Instructor delivered 9 courses in Canberra. Courses were held in locations close to participants' workplaces.

\section{Provide first aid}

This intervention was a 4-hour eLearning PFA course delivered via the Australian Red Cross online portal. The course teaches the fundamental principles, knowledge and skills to provide emergency care for injuries and illnesses in the home or the workplace. Participants received weekly emails for 6 weeks to pace them through the material. Reports from Red Cross were obtained to monitor course progress and flag course completers.

Following online completion, participants in each course were awarded an online 'Certificate of Completion'. For the MHFA courses, participants could undertake an exam (within 3 months of course completion) to become an 'Accredited Mental Health First Aider', valid for 3 years. Staff who completed the eLearning PFA course were offered an option to attend a 1-day assessment (in their own time) to obtain a 'Statement of Attainment' for the Health Training Package, HLTAID003 - Provide First Aid, also valid for 3 years.

\section{Outcomes}

Data were collected at baseline, post training (participants were given 2 months to complete the assessment after they had completed the course), one-year follow-up and two-year follow-up. Only outcomes assessed at baseline and post training are reported here. Data from the one and two- year follow-up questionnaires will be reported in a future publication as data collection is ongoing. Participants were invited to complete an online questionnaire via SurveyMonkey after they had completed the eLearning course, or had attended the face-to-face course. The median length of time between baseline and post training assessment was 85 days.

Initial questions covered sociodemographic information (age, gender, email, daytime telephone number, employment time fraction, marital status, postcode, country of birth, language spoken at home, level of education, Aboriginal and Torres Strait Islander status and whether the respondents managed staff). Subsequent questions centred around a vignette of a person 'John' meeting the DSM criteria for major depression with suicidal thoughts and 'Paula' meeting the DSM criteria for PTSD (previously validated vignettes of two mental disorders which have high prevalence rates and are therefore relatively likely to be encountered in the workplace) $[2,16]$.

\section{Primary outcomes}

These were as follows:

Mental health first aid knowledge This was measured using a 16-item true/false quiz based on the content of the Mental Health First Aid Manual [5]. Respondents were presented with the statements given in Table 1 and asked whether they agreed, disagreed or did not know. The number of correct responses was converted into a percentage. A Pearson correlation coefficient was used to assess test-retest reliability in the control group, which was 0.61 (95\% CI 0.44-0.73).

Desire for social distance This was measured by the Social Distance Scale [17] in relation to the vignettes described above. This scale is widely used, and many studies have shown that it has a single factor structure [18, 19]. Respondents were asked: Would you be happy to: 1 ) To move next door to John/Paula? 2) To spend an evening socializing with John/Paula? 3) To make friends with John/Paula? 4) To work closely with John/Paula on a project at work? 5) To have John/Paula marry into your family? Responses were scored as follows: 1 = Yes definitely (low social distance) to $4=$ Definitely not (high social distance). The internal consistency of the scales in this sample (omega total) was 0.88 for depression and 0.91 for PTSD. Scores were dichotomised at the median of pre- and post-scores. 
Table 1 Mental health first aid knowledge

\begin{tabular}{|c|}
\hline $\begin{array}{l}\text { 1) Half of all people who experience a mental illness have their first } \\
\text { episode by age } 18\end{array}$ \\
\hline $\begin{array}{l}\text { 2) Depressive disorders are the most prevalent mental illness in the } \\
\text { Australian population }\end{array}$ \\
\hline $\begin{array}{l}\text { 3) If a person who is depressed does not want to seek professional } \\
\text { help, it is important to force them to if you can }\end{array}$ \\
\hline 4) Exercise can help relieve depression \\
\hline $\begin{array}{l}\text { 5) Recovery from anxiety disorders requires facing situations which } \\
\text { are anxiety provoking }\end{array}$ \\
\hline $\begin{array}{l}\text { 6) Antidepressant medications can be an effective treatment for } \\
\text { most anxiety disorders }\end{array}$ \\
\hline $\begin{array}{l}\text { 7) When interacting with a person with psychosis, it is best not to } \\
\text { offer them choices of how you can help them because it could } \\
\text { add to their confusion }\end{array}$ \\
\hline $\begin{array}{l}\text { 8) A person with a psychotic illness is less likely to relapse if they } \\
\text { have a good relationship with their family }\end{array}$ \\
\hline $\begin{array}{l}\text { 9) A good way to help a person with a drug or alcohol problem } \\
\text { is to let them know that you strongly disapprove of their } \\
\text { substance use }\end{array}$ \\
\hline $\begin{array}{l}\text { 10) People with mental illnesses are much more likely to be } \\
\text { smokers }\end{array}$ \\
\hline $\begin{array}{l}\text { 11) It is not a good idea to ask someone if they are feeling } \\
\text { suicidal in case you put the idea in their head }\end{array}$ \\
\hline $\begin{array}{l}\text { 12) It is best to get someone having a panic attack to breathe } \\
\text { into a paper bag }\end{array}$ \\
\hline $\begin{array}{l}\text { 13) If someone has a traumatic experience, it is best to make } \\
\text { them talk about it as soon as possible }\end{array}$ \\
\hline 14) It is best not to try to reason with a person having delusions \\
\hline $\begin{array}{l}\text { 15) If a person is intoxicated with alcohol, it is not possible to } \\
\text { make them sober up more quickly by giving them strong } \\
\text { coffee, a cold shower or taking them for a walk }\end{array}$ \\
\hline $\begin{array}{l}\text { 16) If a person becomes unconscious after taking drugs, it is } \\
\text { best to lie them on their side rather than on their back }\end{array}$ \\
\hline
\end{tabular}

\section{Secondary outcomes}

Recognition of mental health problems In order to assess recognition of the problems in the vignettes, participants were asked an open-ended question, "What, if anything do you think is wrong with John/Paula?" For the depression vignette, participants were assessed as having correctly recognised the problem if they mentioned 'depressed' or 'depression' in their response. For the PTSD vignette, responses with any mention of PTSD, post-traumatic stress, or post-traumatic stress disorder were assessed as correct. Pearson correlation coefficients were used to assess test-retest reliability in the control group, which were 0.56 (95\% CI 0.39-0.70) for depression and 0.65 (95\% CI 0.50-0.77) for PTSD.

Beliefs about treatment These were assessed using a 16-item scale based on the 2011 National Survey of Mental Health Literacy and Stigma [20] and a consensus between Australian clinical psychologists, psychiatrists, and GPs established by a national survey [21]. Respondents were presented with sources of potential help for depression and PTSD. For the depression vignette, participants scored 1 point for 'Helpful' responses to each of the following: a typical family GP or doctor; a psychiatrist; a psychologist; becoming more physically active; reading about people with similar problems and how they have dealt with them; psychotherapy; cognitive behaviour therapy; cutting out alcohol altogether; and antidepressants. They also scored 1 point for rating 'dealing with the problem alone' as harmful. For the PTSD vignette, participants scored 1 point for 'Helpful' responses to each of the following: a typical family GP or doctor; a psychiatrist; a psychologist; becoming more physically active; reading about people with similar problems and how they have dealt with them; courses on relaxation, stress management, meditation or yoga; psychotherapy; cognitive behaviour therapy; and receiving information about his problem from a health educator. Depression treatment beliefs ranged from 0 to 10 and PTSD treatment beliefs from 0 to 9. Pearson correlation coefficients were used to assess test-retest reliability in the control group, which were 0.58 (95\% CI 0.40-0.71) for depression and 0.57 (95\% CI 0.40-0.70) for PTSD.

Helping intentions and confidence Intentions to provide help to the person in the vignette were assessed by asking respondents: If John/Paula was a co-worker, I would help him/her. This was scored using a 7-point scale ranging from $1=$ Strongly Disagree to $7=$ Strongly Agree. Responses were coded into two categories: strongly agree/agree vs other. Spearman correlation coefficients were used to assess test-retest reliability in the control group, which were $0.45(0.25-0.61)$ for depression and $0.43(0.23-0.60)$ for PTSD. This was followed with, "Describe all the things you would do to help John/Paula." (open-ended response). Scoring was based on the MHFA Action Plan [22] with quality of intended support ranging from 0 to 12 . Open-ended responses were scored blinded to allocation or occasion. A random sample of 50 responses were double-coded for each vignette, and inter-rater reliability (ICC) was 0.88 for depression and 0.94 for PTSD. Pearson correlation coefficients were used to assess test-retest reliability in the control group, which were 0.18 (95\% CI -0.04, 0.39) for depression and 0.42 (95\% CI 0.21-0.59) for PTSD.

Confidence in providing help to someone at work with depression and PTSD was assessed by asking participants, "How confident do you feel in helping someone at work with a problem like John/Paula?" Confidence was rated using a 5-point scale used in previous studies, $1=$ Not at all to $5=$ Extremely [4]. Pearson correlation coefficients were used to assess test-retest reliability in the control group, which were $0.64(0.49-0.76)$ for depression and $0.50(0.32-0.65)$ for PTSD. 
Personal stigma The Personal Stigma Scale was used to measure participants' stigmatising attitudes to the person described in the vignette [23]. Exploratory Structural Equation Modelling has shown this scale to have factors measuring belief that a person with a mental health problem is weak not sick, and belief that they are dangerous or unpredictable [19]. This scale includes the following 9 items: 1) (John/Paula) could snap out of it if they wanted; 2) (John/Paula)'s problem is a sign of personal weakness; 3$)$ (John/Paula)'s problem is not a real medical illness; 4) (John/Paula) is dangerous; 5) It is best to avoid (John/Paula)'s so that you don't develop this problem yourself; 6) (John/Paula)'s problem makes him/ her unpredictable; 7) You would not tell anyone if you had a problem like (John/Paula)'s; 8) I would not employ someone if I knew they had a problem like (John/ Paula)'s; 9) I would not vote for a politician if I knew they had suffered a problem like (John/Paula)'s. Scoring ranged from $1=$ Strongly Agree to $5=$ Strongly Disagree. The Omega coefficient in this sample for' Weak not sick' stigma was 0.83 for depression and 0.82 for PTSD. For Dangerous/unpredictable stigma, the Omega coefficient was 0.70 for depression and 0.72 for PTSD. 'Weak not sick' scores showed substantial negative skew so were dichotomised at the median, which was the highest possible score (5) for both depression and PTSD vignettes.

Course satisfaction Course satisfaction was assessed using the following questions: 1) How much of the training did you complete? $(1=$ none of it, $2=$ part of it, $3=$ most of it, $4=$ all of it); 2) How easy was the material to understand? ( $1=$ very easy, $2=$ easy, $3=$ neither easy nor difficult, $4=$ difficult, 5 = very difficult);

3) How much did you learn from the course? $(1=\mathrm{a}$ great deal, $2=\mathrm{a}$ fair bit, $3=$ not very much, $4=$ almost nothing); 4) How useful was the course? ( $1=$ very useful, $2=$ useful, $3=$ not very useful, $4=$ not at all useful); 5 ) Do you think you will use the training material in the future? ( $1=$ yes, $2=$ no, $3=$ not sure); 6) Would you recommend the course to others? $(1=$ yes definitely, 2 = probably, 3 = probably not, $4=$ definitely not); 7) What did you like about the training materials? 8) What did you dislike about the training materials? These questions were asked post-course only.

\section{Sample size estimation}

In order to calculate the required sample size, we considered the main hypothesis of interest to be the following: that blended MHFA training would be superior to MHFA eLearning in achieving improvements in MHFA knowledge and reductions in desire for social distance. The sample size required to detect differences between these two modes of training was larger than that required to detect differences between these modes and PFA training. Consequently, we chose a small effect size to evaluate the difference between the two modes of MHFA training, as an effect size smaller than this may not be meaningful in terms of participant outcomes. According to Stata Release 12, 165 participants were required per group. For a repeated measures design, with 1 pre-training measure and 3 post-training measures, using the change method of sample size calculation and assuming a conservative 0.70 correlation between preand post-measurements (based on [24]), to detect an effect size of Cohen's $\mathrm{d}=0.20$ (or $\mathrm{h}=0.20$ ), with a power $=0.80$ and an alpha $=0.05$, Increasing the sample size by $20 \%$ to account for attrition, the total sample size required was estimated to be 594 (198 participants per group).

\section{Adverse events}

In the event that a participant felt distressed during survey completion or while undertaking the training, a list of contacts was included at the end of each online survey. This included phone numbers for Lifeline, Suicideline (Victoria only), SANE, Emergency Services (000) and relevant EAP providers. Lifeline's Online Crisis Chat link was also included. Participants were encouraged to contact the trial manager to report any adverse events. None were reported.

\section{Ethics}

The study was approved by the University of Melbourne Human Ethics Sub-Committee (Ethics ID 1341345.2).

\section{Statistical analysis}

An intention-to-treat approach was used, with all participants included in the analyses. Data were analysed using mixed-effects models for continuous and binary outcome variables, with group-by-measurement occasion interactions. This method takes into account the hierarchical structure of the data in the analysis of differences between the groups, i.e. the correlation of measurement occasions within participants. It can produce unbiased estimates when a proportion of the participants withdraw before the completion of the study, based on the reasonable assumption that these data are missing at random [25]. As tertiary-educated participants were somewhat more likely to have data at post (OR $=1.39,95 \%$ CI $0.99-1.95, p=.057$ ), education was included as a fixed effect in order to help meet the missing at random assumption. For outcome measures with no substantial baseline imbalance, effect sizes (Cohen's d) were calculated by dividing the difference between the two group means at post-training by their pooled standard deviation. With baseline imbalances, Cohen's $d$ was calculated by dividing the mean change in each condition by the pooled standard 
deviation post-training. Analyses were performed in Stata 14 and RStudio and the significance level was set at $p<.05$.

\section{Results}

\section{Participant flow and numbers analysed}

The CONSORT flow diagram of the number of participants at each stage of the trial is given in Fig. 1. All the participants included in the analyses completed the first questionnaire and 319 (52.5\%) participants did not complete the post-test questionnaire. The sole predictor of having data at post-test was assignment to the PFA group $(\mathrm{OR}=0.59,95 \% \mathrm{CI} 0.39-0.87, p=0.008)$. The three groups were similar in baseline sociodemographic characteristics, indicating that randomisation resulted in comparable groups (see Table 2).

\section{Participants' characteristics}

The mean age of participants was 41.2 years $(\mathrm{SD}=10.9$ ), 74.1\% were female, $87.1 \%$ spoke English at home, $66.1 \%$ were tertiary educated, $1.2 \%$ were Aboriginal or Torres Strait Islander, $65.4 \%$ were married or in de facto relationships and $31.2 \%$ managed staff (See Table 2).

\section{Program use}

In the MHFA eLearning group, $68.8 \%$ attempted the online course compared to $73.4 \%$ of those in the blended MHFA group and $73.3 \%$ of those in the PFA eLearning group. In the MHFA eLearning group, 55.3\% completed the course compared to $64.8 \%$ of those in the blended MHFA group and $43.3 \%$ of those in the PFA eLearning group, the only difference that reached statistical significance $(p<0.001)$. The MHFA course website automatically captured when each module was completed. The mean number of modules completed (among those who attempted the course) was $4.19(\mathrm{SD}=1.75)$ for MHFA eLearning and $4.53(\mathrm{SD}=1.38)$ for the blended group. Among all participants these means were $2.88(\mathrm{SD}=2.42)$ and $3.32(\mathrm{SD}=2.33)$ respectively. In the blended group, $59.8 \%$ attended face-to-face training and $58.8 \%$ attended this and also completed all five modules. The website captured first and last access of the online courses with timestamps. Among participants in the two MHFA groups, $29.2 \%$ spent less than one hour on the course, $21.4 \%$ spent $1-2 \mathrm{~h}, 16.3 \%$ spent $2-8 \mathrm{~h}$ and $33.1 \%$ spent more than $8 \mathrm{~h}$.

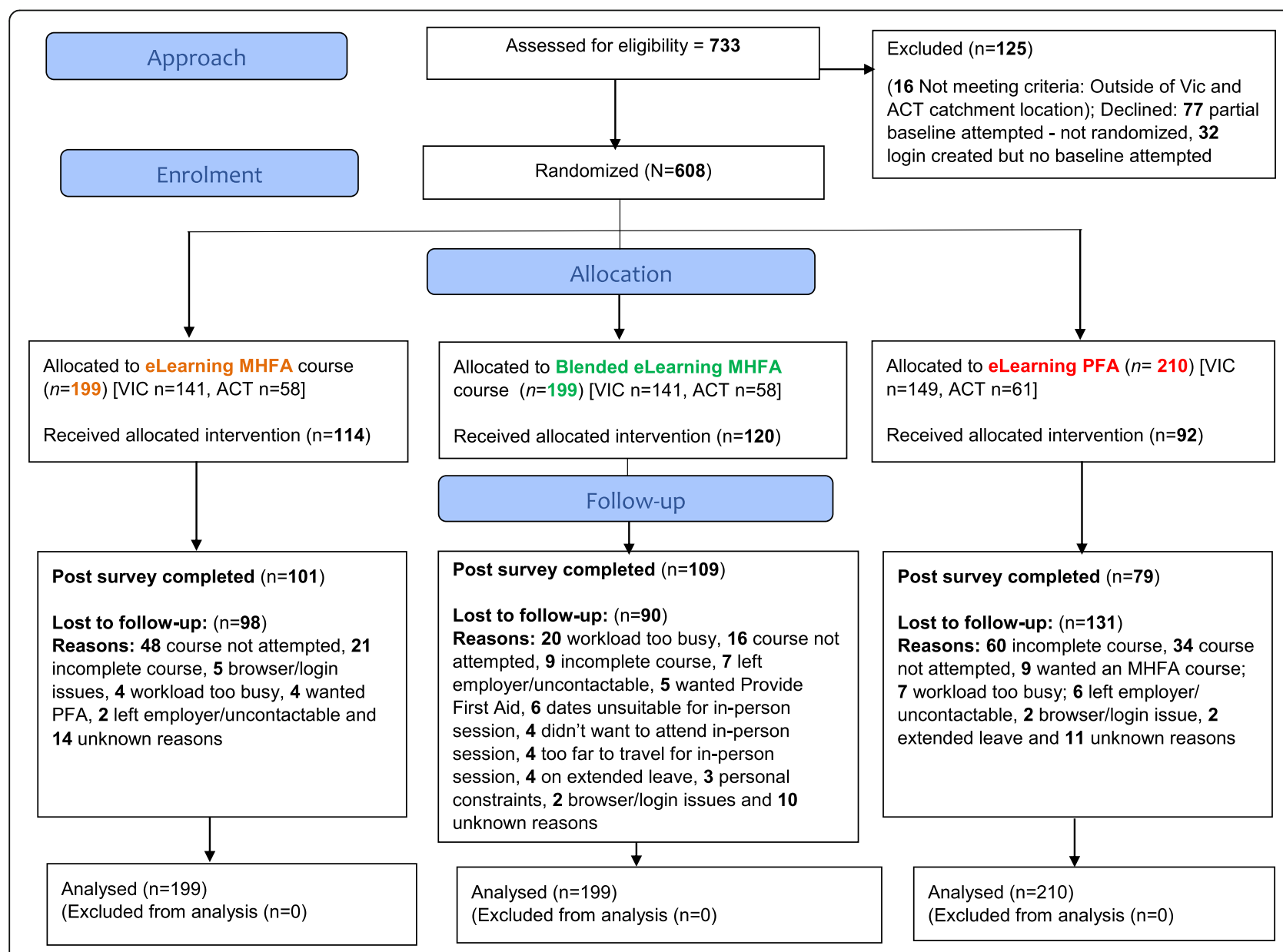

Fig. 1 CONSORT flow diagram for the study 
Table 2 Baseline characteristics of participants in intervention and control groups

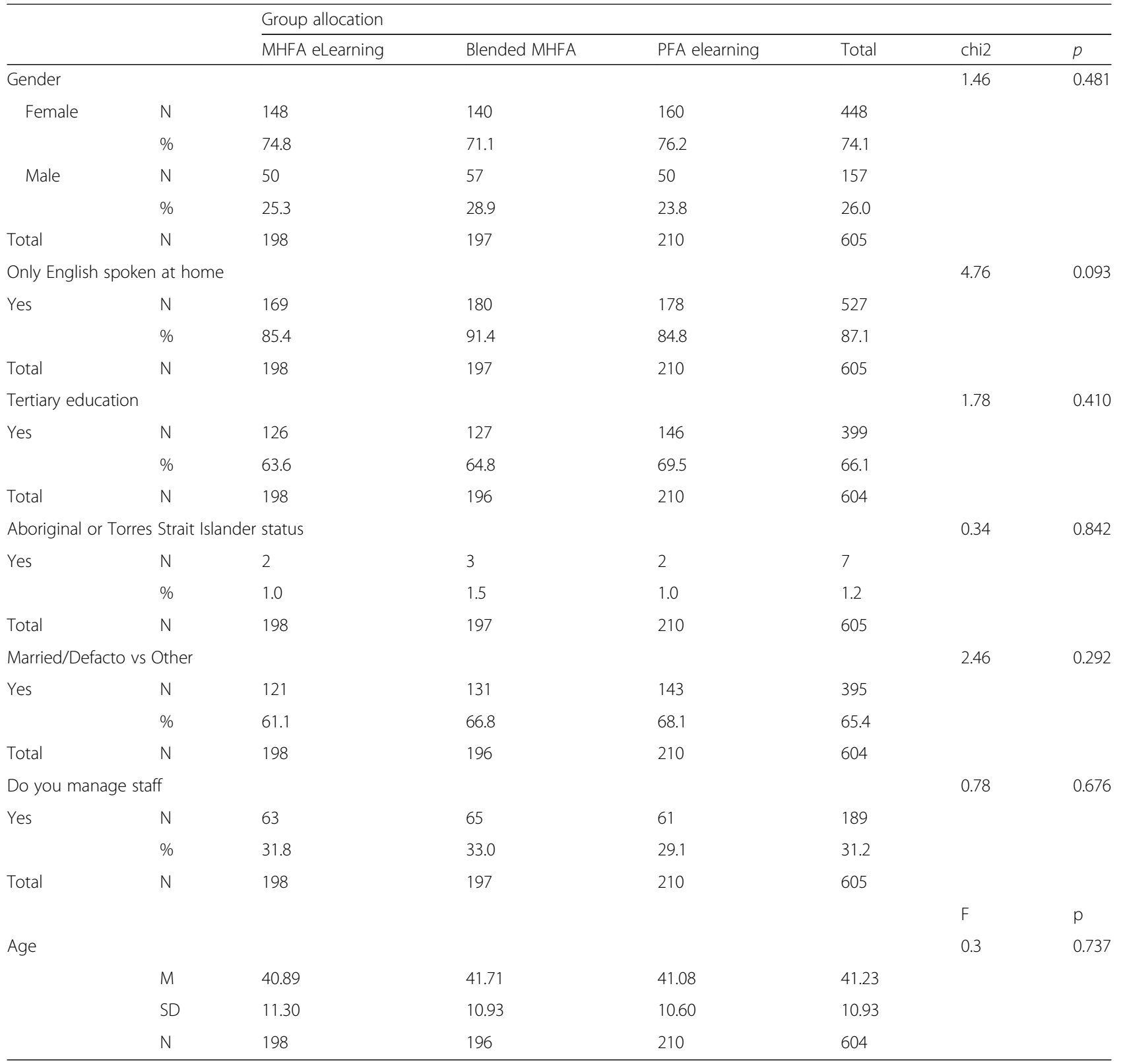

\section{Primary outcomes}

Baseline and post scores on all outcome measures are presented in Table 3, which also presents the results from the planned contrasts, estimating the mean difference in change over time between groups.

There were significantly greater improvements in knowledge in both MHFA eLearning and blended MHFA groups compared to PFA eLearning. These differences were greater than large in size $(d=1.35$ and $\mathrm{d}=1.55$ respectively). The difference between MHFA eLearning and blended MHFA was not significant.

Those in the eLearning MHFA group were significantly more likely to show a reduced desire for social distance from a person with depression or PTSD than those in the PFA eLearning group. The reduction in social distance in the blended group was not significantly greater than the PFA eLearning group. There were no significant differences between the MHFA eLearning and blended courses.

\section{Secondary outcomes}

Recognition of mental disorders

For the depression vignette, correct recognition was high at baseline and there was no difference in change in recognition between MHFA eLearning and PFA eLearning. There were significantly greater improvements in the 


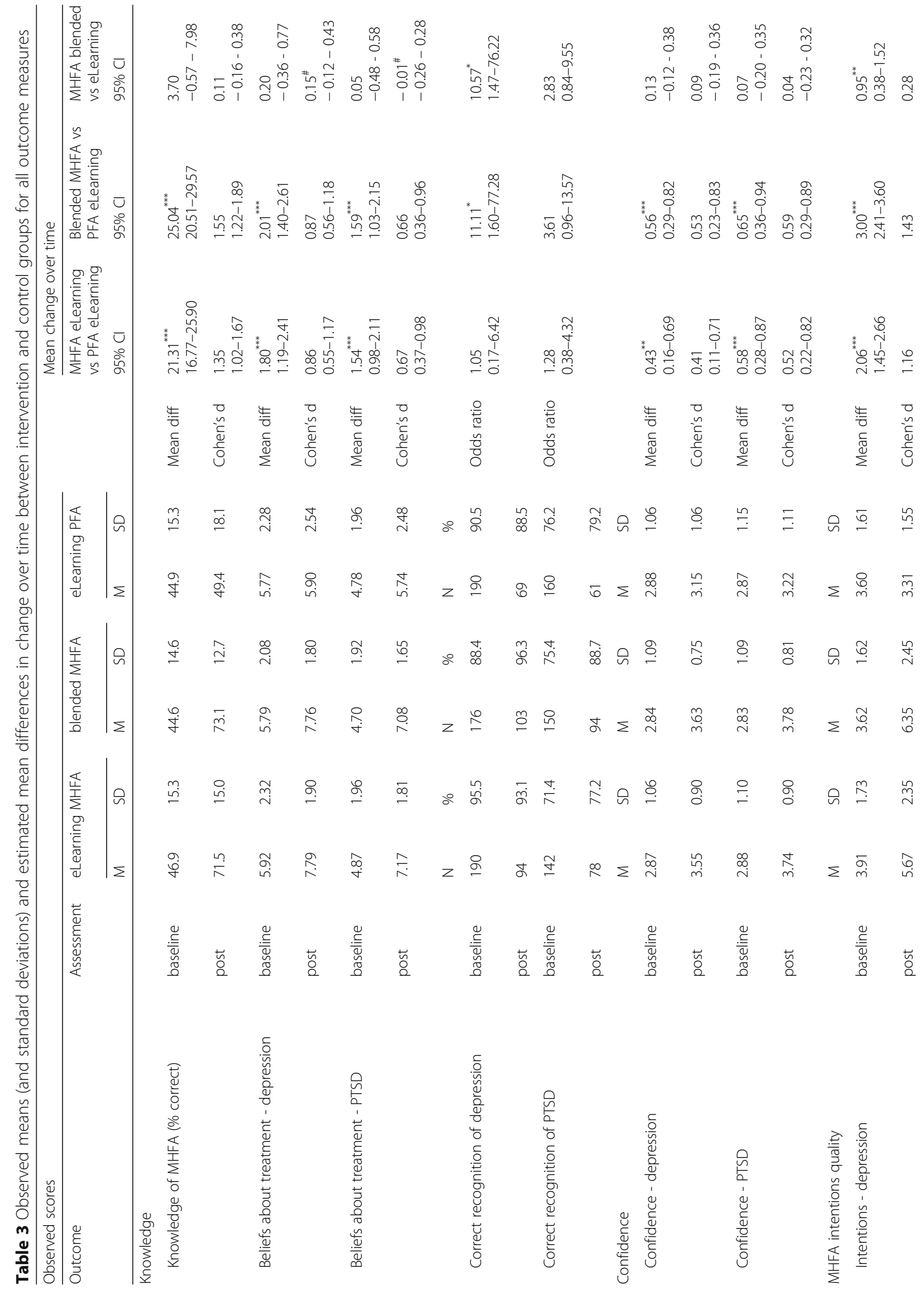


Reavley et al. BMC Psychiatry (2018) 18:312

Page 10 of 14

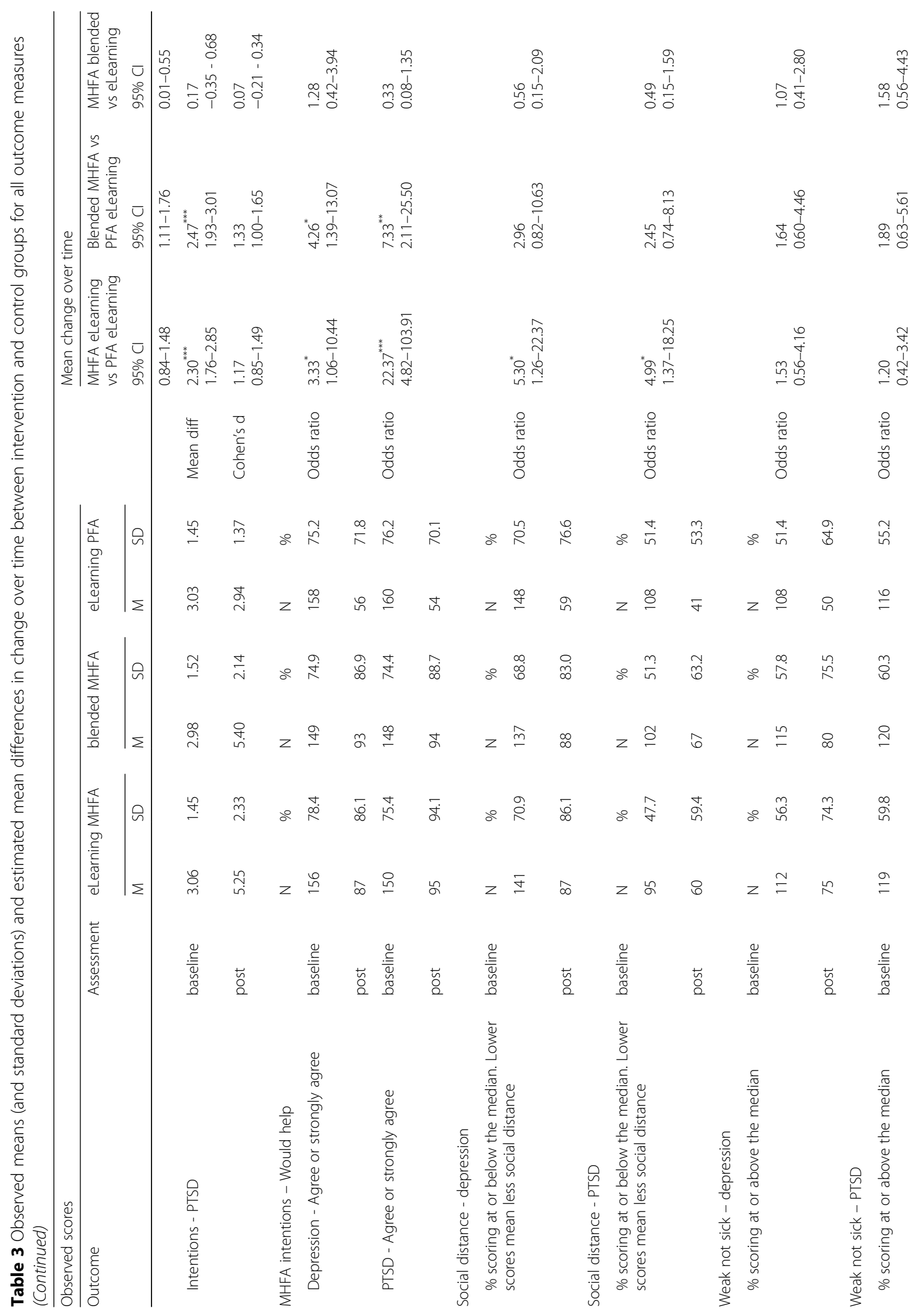




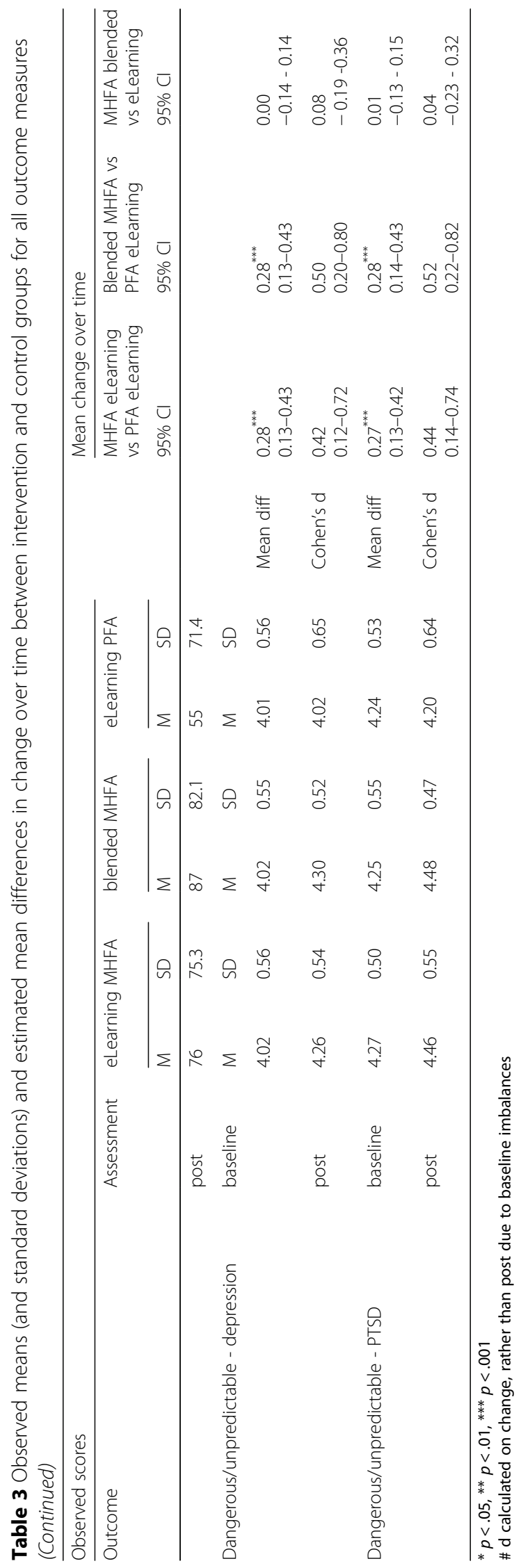


blended MHFA vs PFA eLearning groups and in the blended MHFA vs MHFA eLearning groups. For PTSD, changes were not significantly different between groups.

\section{Beliefs about treatment}

Beliefs about treatment for depression and PTSD moved closer to those of health professionals in both the MHFA eLearning and blended MHFA groups compared to PFA eLearning. These differences were large in size for depression ( $\mathrm{d}=0.86$ and $\mathrm{d}=0.87$ respectively) and medium in size for PTSD ( $d=0.67$ and $d=0.66$ respectively). The differences between MHFA eLearning and blended MHFA were not significant.

\section{Quality of helping intentions and confidence}

There were greater increases in agreement with intentions to help a person with depression and PTSD in both the MHFA eLearning and blended MHFA groups compared to PFA eLearning. The differences between MHFA eLearning and blended MHFA were not significant.

There were greater improvements in quality of helping intentions for depression and PTSD in both the MHFA eLearning and blended MHFA groups compared to PFA eLearning. These differences were large in size for depression $(\mathrm{d}=1.16$ and $\mathrm{d}=1.43$ respectively) and PTSD $(\mathrm{d}=1.17$ and $\mathrm{d}=1.33$ respectively). There were also significantly greater improvements (of small effect size) in the blended MHFA group than in the eLearning MHFA group for depression $(\mathrm{d}=0.28)$, although not for PTSD.

There were greater improvements in confidence for depression and PTSD in both the MHFA eLearning and blended MHFA groups compared to PFA eLearning. These differences were medium in size for depression $(\mathrm{d}=0.41$ and $\mathrm{d}=0.53$ respectively) and PTSD $(\mathrm{d}=0.52$ and $\mathrm{d}=0.59$ respectively). The differences between MHFA eLearning and blended MHFA were not significant.

\section{Personal stigma}

Belief that people with depression or PTSD are 'weak not sick' was low at baseline, with no significant differences in improvement between courses. For beliefs in dangerousness/unpredictability, those in the blended and eLearning MHFA groups were significantly more likely to show reduced stigma towards people with depression and PTSD than those in the PFA eLearning group. There were no significant differences between the MHFA eLearning and blended courses.

\section{Course satisfaction}

Over $90 \%$ of participants in all groups reported completing all the course and over $75 \%$ of participants in all groups reported finding the material very easy or easy to understand. However, it is interesting to note that the self-reported data differs from the objective data captured from the website, which suggested lower completion rates. Differences between groups did not reach statistical significance. When asked how much they had learnt from the course, $61.5 \%$ of participants in blended MHFA reported learning a great deal, compared to $36.6 \%$ in the eLearning course and $30.4 \%$ in the PFA course. Similarly, $72.5 \%$ of participants in the blended course reported finding it very useful, compared to $57.4 \%$ in the MHFA eLearning group and $44.3 \%$ in the PFA group. When asked if they would use the training material in future, $89.1 \%$ of those in the eLearning group, $89.0 \%$ of those in the blended MHFA group and $73.4 \%$ of those in the PFA group reported that they would. When asked if they would recommend the course to others, $72.3 \%$ of those in the eLearning group, $83.5 \%$ of those in the blended MHFA group and $62 \%$ of those in the PFA group reported that they definitely would. Differences between groups on these last four questions all reached statistical significance at $p<0.05$ level or lower.

\section{Discussion}

Both the eLearning MHFA and blended MHFA courses had positive effects compared to PFA eLearning on the primary outcome of mental health first aid knowledge and the secondary outcomes of beliefs about professional treatments, quality of helping intentions and confidence in helping and personal stigma (related to dangerousness and unpredictability) towards a person with depression or PTSD. Thus, the first hypothesis was mostly supported. These findings are consistent with the growing number of studies demonstrating the effectiveness of MHFA courses [7], including two RCTs, one of which was conducted in Australian employees [12] and the other, more recently, in UK medical students [26].

In this study, the effects were similar for the eLearning and blended modes of MHFA courses, with most outcomes showing very small non-significant differences between the modes. There were some signs of blended MHFA being superior to eLearning MHFA, with significantly greater improvements in recognition of depression and quality of intentions to help a person with depression, although not for PTSD. Thus, the second hypothesis was mostly not supported.

This study makes a valuable contribution to the literature on the impact of modes of learning, which until now, has largely focused on students enrolled in formal education, who may be expected to have higher levels of motivation for attending and completing online courses $[13,14]$. Poor retention rates in online studies are common and a key aim of the trial was to explore whether the addition of a face-to-face component was worthwhile in terms of learner outcomes in the MHFA course. It is 
notable that participants in the blended MHFA group were significantly more likely to complete the online course $(64.8 \%$ vs $55.3 \%)$ and there were also trends towards a higher number of completed modules and more time spent on the course overall among participants in that group. Participants were also more likely to rate the blended MHFA course highly in terms of usefulness, amount learned and intentions to recommend to others, thus partly supporting the third hypothesis. It is therefore possible that blended MHFA may lead to improved behavioural changes in the longer term, such as a greater likelihood of participants providing more appropriate help to a person who develops a mental health problem. Data being collected at 1- and 2-year follow-up may shed more light on this, potentially adding to a sparse literature in the area of longer-term impacts of such training.

Additionally, one third of participants spent less than two hours online in total, despite being given permission to do the training in work time and the convenience of being able to $\log$ in as often as they wished and on more than one computer. Several factors may have contributed to the lower than expected usage, including a Victorian state government election, a department restructure (which led to email address changes) and the fact that the department-supplied internet browser was not optimal for the eLearning program. Such implementation difficulties are often seen in workplace-based interventions and complicate efforts to evaluate programs and to more widely disseminate those that are effective $[27,28]$.

Greater increases in depression recognition and helping intentions in the blended MHFA group may have arisen due to the in-depth discussion and mental health first aid skills practice that took place in the face-to-face component of the blended MHFA course. For example, in the blended MHFA course, there were three additional case studies, and a role play where participants practiced implementing the MHFA action plan and could then debrief with other participants and their instructor.

Strengths of the study include the use of a control intervention closely matched to the MHFA courses in time commitment and mode of delivery, while limitations include the larger than expected attrition and consequent lack of power to assess differences between the two modes of MHFA delivery. Furthermore, the majority of participants missing at post-test were those who did not complete their assigned course. An additional limitation is the fact that intentions may not translate into actual behaviours, although there is some evidence that mental health first aid intentions at baseline are associated with actual behaviour at follow-up [29, 30]. As part of the current study, data on actual behaviours will be collected at 1- and 2-year follow-up. Future studies could incorporate role plays using simulated situations to evaluate the impact of MHFA training on participant skills, although such methods may be better suited to training of health professionals than members of the public [31].

\section{Conclusions}

Both blended and eLearning MHFA courses are more effective in improving knowledge, attitudes and behavioural intentions than a control intervention. However, the blended MHFA course was minimally more effective than the eLearning MHFA course in improving recognition of depression and quality of intentions to help a person with depression Course satisfaction ratings were higher in those in the blended course, potentially leading to greater benefits in the future. Longer-term follow-up is needed to explore this.

\section{Abbreviations \\ ACT: Australian Capital Territory; EAP: Employee Assistance Program; MHFA: Mental Health First Aid; OR: Odds ratio; PFA: Provide First Aid; PTSD: Post-traumatic stress disorder; RCT: Randomised controlled trial; SD: Standard deviation; VIC: Victoria}

\section{Acknowledgements \\ We would like to thank Anna Ross for assistance with coding open-ended responses and Stefan Cvetkovski for his assistance with the original analysis plan. \\ Funding \\ The trial was funded by the National Health and Medical Research Council (APP1061636), which played no role in the design of the study, data collection, analysis, interpretation of data or writing of the manuscript. AFJ and NJR are supported by National Health and Medical Research Council Fellowships.}

Availability of data and materials

Requests for access to the data should be made to the first author.

\section{Authors' contributions}

AFJ, NJR, BK and JF contributed to the development and management of the project. BK and NB delivered MHFA face-to-face training. AJM carried out the data analysis. NJR drafted the manuscript and all other authors suggested improvements. All authors read and approved the final manuscript.

Ethics approval and consent to participate

The study was approved by the University of Melbourne Human Research Ethics Committee (Ethics ID 1341345.2). Participants gave online consent to participate.

Consent for publication

Not applicable.

\section{Competing interests}

AFJ and BK are the co-founders of MHFA training and Chair of the Board of MHFA International. NB is the current CEO of MHFA International. NJR and JF has no competing interests. AJM is currently acting as an Associate Editor for BMC Psychiatry.

\section{Publisher's Note}

Springer Nature remains neutral with regard to jurisdictional claims in published maps and institutional affiliations. 


\section{Author details}

Centre for Mental Health, Melbourne School of Population and Global Health, University of Melbourne, 207 Bouverie Street, Carlton, VIC 3010, Australia. ${ }^{2}$ Mental Health First Aid Australia, 369 Royal Parade, Parkville, VIC 3052, Australia.

Received: 26 April 2018 Accepted: 17 September 2018

Published online: 26 September 2018

\section{References}

1. Reavley NJ, Jorm AF. Willingness to disclose a mental disorder and knowledge of disorders in others: changes in Australia over 16 years. Aust N Z J Psychiatry. 2014:48(2):162-8.

2. Slade T, Johnston A, Oakley Browne MA, Andrews G, Whiteford H. 2007 National Survey of mental health and wellbeing: methods and key findings. Aust N Z J Psychiatry. 2009;43(7):594-605.

3. Kitchener BA, Jorm AF, Kelly CM. mental health first aid international manual. Melbourne: Mental Health First Aid International; 2015.

4. Kitchener BA, Jorm AF. Mental health first aid training for the public: evaluation of effects on knowledge, attitudes and helping behavior. BMC Psychiatry. 2002;2:10

5. Kitchener B, Jorm AF, Kelly C. Mental health first aid manual. 4th ed. Mental Health First Aid Australia: Melbourne; 2017.

6. Jorm AF, Kitchener BA: The truth about mental health first aid training Psychiatr Serv 2018, 169(4):492.

7. Morgan AJ, Ross AM, Reavley NJ. Systematic review and meta-analysis of mental health first aid training: effects on knowledge, stigma, and helping behaviour. under review. 2018

8. Sanders D, Crowe S. Overview of health promotion in the workplace. In: Scriven A, Orme J, editors. Health Professional Perspectives. London: Macmillan; 1996 p. 199-209.

9. Martin A, Sanderson K, Cocker F. Meta-analysis of the effects of health promotion intervention in the workplace on depression and anxiety symptoms. Scand J Work Environ Health. 2009;35(1):7-18

10. Kitchener BA, Jorm AF. Mental health first aid training in a workplace setting: a randomized controlled trial [ISRCTN13249129]. BMC Psychiatry. 2004:4:23.

11. Wan Mohd Yunus WMA, Musiat P, Brown JSL. Systematic review of universal and targeted workplace interventions for depression. Occup Environ Med. 2018;75(1):66-75

12. Jorm AF, Kitchener BA, Fischer JA, Cvetkovski S. Mental health first aid training by e-learning: a randomized controlled trial. Aust N Z J Psychiatry. 2010;44(12):1072-81.

13. Liu Q, Peng W, Zhang F, Hu R, Li Y, Yan W. The effectiveness of blended learning in health professions: systematic review and meta-analysis. J Med Internet Res. 2016;18(1):e2.

14. Sitzmann T, Kraiger $K$, Stewart DB, Wisher R. The comparative effectiveness of web-based and classroom instruction: a meta-analysis. Pers Psychol. 2006; 59:623-64.

15. Griffiths KM, Carron-Arthur B, Parsons A, Reid R. Effectiveness of programs for reducing the stigma associated with mental disorders. A meta-analysis of randomized controlled trials. World Psychiatry. 2014;13(2):161-75.

16. Reavley NJ, Jorm AF. Stigmatizing attitudes towards people with mental disorders: findings from an Australian National Survey of mental health literacy and stigma. Aust N Z J Psychiatry. 2011:48(12):1086-93.

17. Link BG, Phelan JC, Bresnahan M, Stueve A, Pescosolido BA. Public conceptions of mental illness: labels, causes, dangerousness, and social distance. Am J Public Health. 1999;89(9):1328-33.

18. Jorm AF, Oh E. Desire for social distance from people with mental disorders. Aust N Z J Psychiatry. 2009;43(3):183-200.

19. Yap MBH, Mackinnon AJ, Reavley NJ, Jorm AF. The measurement properties of stigmatising attitudes towards mental disorders: results from two community surveys. Int J Methods Psychiatr Res. 2014;23(1):49-61.

20. Reavley NJ, Morgan AJ, Jorm AF. Development of scales to assess mental health literacy relating to recognition of and interventions for depression, anxiety disorders and schizophrenia/psychosis. Aust N Z J Psychiatry. 2014 48(1):61-9.

21. Morgan AJ, Jorm AF, Reavley NJ. Beliefs of Australian health professionals about the helpfulness of interventions for mental disorders: differences between professions and change over time. Aust N Z J Psychiatry. 2013; 47(9):840-8
22. Rossetto A, Jorm AF, Reavley NJ. Quality of helping behaviours of members of the public towards a person with a mental illness: a descriptive analysis of data from an Australian national survey. Ann General Psychiatry. 2014;13(1):2

23. Griffiths KM, Christensen H, Jorm AF, Evans K, Groves C. Effect of web-based depression literacy and cognitive-behavioural therapy interventions on stigmatising attitudes to depression: randomised controlled trial. $\mathrm{Br} J$ Psychiatry. 2004;185:342-9.

24. Means B, Toyama Y, Murphy R, Baki M. The Effectiveness of Online and Blended Learning: A Meta-Analysis of the Empirical Literature. Teach Coll Rec. 2010;115(030303).

25. Rabe-Hesketh S, Skrondal A. Classical latent variable models for medical research. Stat Methods Med Res. 2008;17(1):5-32

26. Davies EB, Beever E, Glazebrook C. A pilot randomised controlled study of the mental health first aid eLearning course with UK medical students. BMC Med Educ. 2018;18(1):45.

27. Montano D, Hoven $\mathrm{H}$, Siegrist J. Effects of organisational-level interventions at work on employees' health: a systematic review. BMC Public Health. 2014;14:135.

28. Olsen O, Albertsen K, Nielsen ML, Poulsen KB, Gron SM, Brunnberg HL. Workplace restructurings in intervention studies - a challenge for design, analysis and interpretation. BMC Med Res Methodol. 2008:8:39.

29. Yap MBH, Jorm AF. Young people's mental health first aid intentions and beliefs prospectively predict their actions: findings from an Australian National Survey of youth. Psychiatry Res. 2012;196(2-3):315-9.

30. Rossetto A, Jorm AF, Reavley NJ. Predictors of adults' helping intentions and behaviours towards a person with a mental illness: a six-month follow-up study. Psychiatry Res. 2016;240:170-6.

31. Boukouvalas EA, El-Den S, Chen TF, Moles R, Saini B, Bell A, O'Reilly CL. Confidence and attitudes of pharmacy students towards suicidal crises: patient simulation using people with a lived experience. Soc Psychiatry Psychiatr Epidemiol. 2018.

Ready to submit your research? Choose BMC and benefit from:

- fast, convenient online submission

- thorough peer review by experienced researchers in your field

- rapid publication on acceptance

- support for research data, including large and complex data types

- gold Open Access which fosters wider collaboration and increased citations

- maximum visibility for your research: over $100 \mathrm{M}$ website views per year

At BMC, research is always in progress.

Learn more biomedcentral.com/submission 